\title{
Luminosity Reduction Caused by Phase Modulations at the HL-LHC Crab Cavities
}

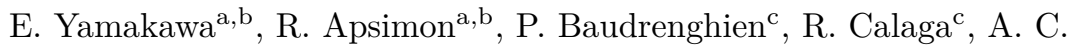 \\ Dexter $^{\mathrm{a}, \mathrm{b}}$ \\ ${ }_{5}{ }^{a}$ Engineering Department, University of Lancaster, Lancaster LA1 4YW, United Kingdom \\ ${ }^{b}$ The Cockcroft Institute, SciTech Daresbury, Daresbury, Warrington, United Kingdom \\ ${ }^{c}$ CERN, 1211 Geneva, Switzerland
}

\begin{abstract}
The design of the High-Luminosity Large Hadron Collider (HL-LHC) requires two pairs of crab cavities to be installed either side of Interaction Points (IPs) 1 (ATLAS) and 5 (CMS) to compensate for the geometric reduction in luminosity due to the beam crossing angle at the IP. The HL-LHC beam current is a factor of two larger than the LHC design value. The existing RF system has insufficient power to use the existing low level RF (LLRF) scheme for HL-LHC and therefore a new scheme is proposed which results in an irregular bunch pattern in the ring; here in referred to as a phase modulation. In this paper we study the effect of this phase modulation on the crab cavity scheme and the resulting impact on peak luminosity. We have developed an analytical model to calculate the luminosity and its dependence on the related beam and RF parameters. We compare this model to tracking simulations in PYTRACK and show a good agreement between the model and simulations. In the case of a coherent phase error between the counter-rotating bunch trains, having the maximum expected time shift of $100 \mathrm{ps}$ ( 0.25 radians at the $\mathrm{RF}$ frequency), the reduction of analytical peak luminosity is found to be $1.89 \%$ when the crabbing voltage is $6.8 \mathrm{MV}$. For incoherent phase errors, the luminosity reduction for a 100 ps phase error is $5.67 \%$; however the expected incoherent phase error is significantly less than $100 \mathrm{ps}$. These reductions are not foreseen as an issue when the crabbing scheme is used for luminosity levelling during physics experiments.
\end{abstract} Keywords: Crab cavity, Luminosity, HL-LHC, LLRF

Preprint submitted to Elsevier

August 23, 2018 


\section{Introduction}

The HL-LHC project due to receive first beams in 2026, aims to increase the luminosity of the LHC from its current design value of $1 \times 10^{34} \mathrm{~cm}^{-2} \mathrm{~s}^{-1}$ by a factor of five for the nominal scenario [1. Key features of the luminosity upgrade are the reduced transverse beam size and the increased crossing angle introduced to mitigate long-range beam-beam effects [2]. The design crossing angle at ATLAS and CMS (IPs 1 and 5 respectively) is 285 urad and operation prior to 2018 has typically been close to this value [3]. The HL-LHC has an increased crossing angle of around $500 \mu \mathrm{rad}$ [1]. Version 1.3 of the HL-LHC baseline optical parameters for collisions with $\beta^{*}$ near $0.15 \mathrm{~m}$ [1] are summarized in Table 1 , where $\beta^{*}$ is defined as the value of the $\beta$-function at the IP.

The overlap density of the colliding bunches is reduced with the presence of a crossing angle as shown in Figure 1. The resulting luminosity loss is expressed by the Piwinski reduction factor $(R)$;

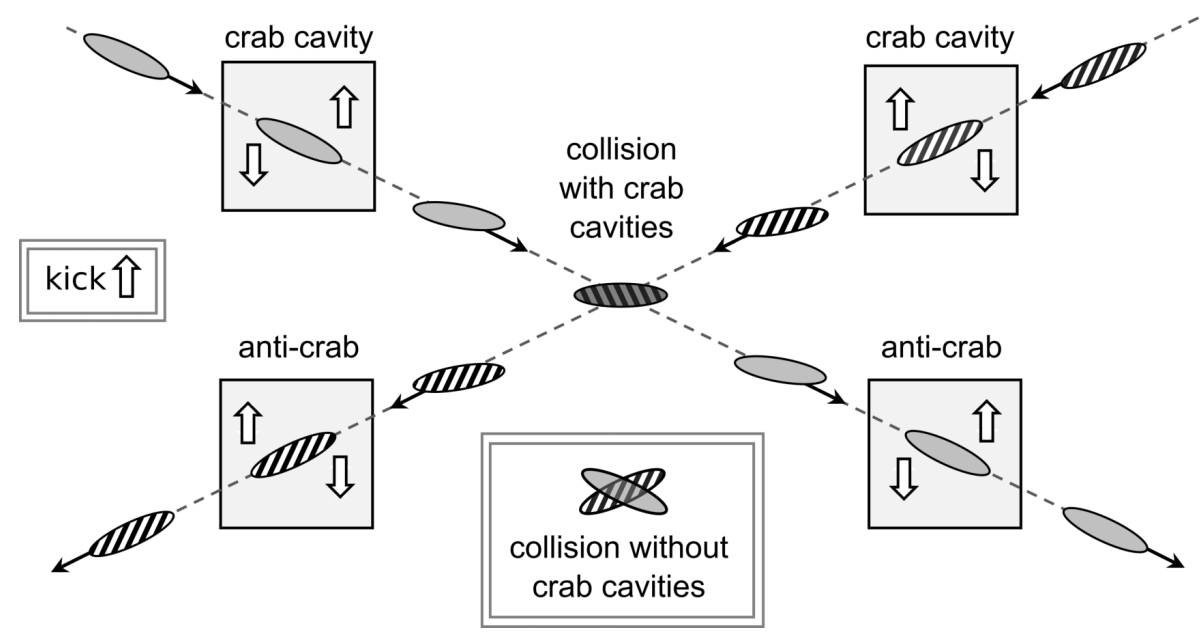

Figure 1: Simplified diagram of the collisions at the IP with and without crab cavities. There is a $\pi$ phase advance between crab and anti-crab cavities. 
Table 1: List of parameters for the baseline optics (HL-LHCV1.3).

\begin{tabular}{l|r}
\hline Machine circumference & $26659 \mathrm{~m}$ \\
Proton energy at collision & $7 \mathrm{TeV}$ \\
Beam intensity $N$ & $2.2 \times 10^{11} p p b$ \\
Number of bunch $n_{b}$ & 2748 \\
r.m.s bunch length $\left(\sigma_{z}\right)$ & $9.0 \mathrm{~cm}$ \\
Bunch spacing & $25 \mathrm{~ns}$ \\
Longitudinal emittance & $3.03 \mathrm{eVs}$ \\
Harmonic number & 35640 \\
Transition gamma & 55.76 \\
Transverse normalized emittance $\epsilon_{n(x, y)}($ r.m.s $)$ & $2.5 \mathrm{\mu m}$ \\
Revolution frequency & $11.2455 \mathrm{kHz}$ \\
Synchrotron frequency & $23.8 \mathrm{~Hz}$ \\
RF frequency of main cavity & $400.79 \mathrm{MHz}$ \\
Total RF voltage of main cavity & $16 \mathrm{MV}$ \\
Full crossing angle & $480 \mathrm{\mu rad}$ \\
Crab cavity voltage & $3.4 \mathrm{MV} / \mathrm{cavity}$ \\
Crab cavity RF frequency & $400.79 \mathrm{MHz}$ \\
\hline
\end{tabular}

$$
R(\theta)=\frac{1}{\sqrt{1+\left(\frac{\sigma_{z}}{\sigma_{x}^{*}} \frac{\theta}{2}\right)^{2}}},
$$

where $\sigma_{x}^{*}$ is the transverse beam size at the IP, $\sigma_{z}$ is the r.m.s bunch length and $\theta$ is the full crossing angle.

Crab cavities are RF deflectors, phased so that the nominal bunch centroid receives no kick, while the head and tail receive transverse kicks in opposite directions, to rotate the bunch envelope. In order to recover the geometric overlap at the IPs in the HL-LHC, superconducting crab cavities [4] will be installed both up- and down-stream of IPs 1 and 5, partially compensating the geometric luminosity reduction at the IPs (Figure 1). A full compensation is not 
required as this would result in the peak luminosity being too high and excessive pile up for the experiment. A partial compensation allows for a constant peak luminosity during physics runs by using a luminosity leveling scheme [7] 9 . The crab cavities are being first tested in CERN's Super Proton Synchrotron (SPS) prior to installation in the LHC.

The HL-LHC project plans to use a local crabbing scheme [10] to create local crabbing bumps around the IPs. In this scheme, the crab cavities after the IP are situated at a betatron phase advance, $\mu=\pi$, downstream of the first set of crab cavities, and the Twiss parameters, $\alpha$ and $\beta$ are the same, thus cancelling the effect of the upstream crab cavities; the downstream crab cavities will be referred to here as anti-crab cavities.

The planned scheme requires two cavities to achieve the required crabbing voltage $V_{1}$ before the IP and another two for the required anti-crabbing voltage ${ }_{45} \quad V_{2}$ after the IP (for two beams and two IPs sixteen cavities in total are required). The total crabbing voltage required for full compensation at the IP [11] is given as

$$
V_{1}=\frac{c E_{s} \tan \theta / 2}{e \omega \sqrt{\beta^{*} \beta_{c . c}} \sin \mu},
$$

where $c$ is the speed of light, $E_{s}$ is the energy of the synchronous particle, $e$ is the charge of the proton, $\omega$ is the angular frequency of the crab cavity RF, $\mu$ is ${ }_{50}$ the betatron phase advance between the upstream crab cavity and the IP, $\beta^{*}$ and $\beta_{c . c}$ are the $\beta$ functions at the IP and crab cavity locations respectively. The crab cavities are to be installed at locations where $\beta_{c . c}$ is large to reduce the required cavity voltages as determined by Eq. 2. The phase advance from the crab cavity to the IP and the IP to the anti-crab cavity are set as $\mu=\pi / 2$. The required total anti-crab voltage after the IP is given by

$$
V_{2}=-R_{22} V_{1}
$$

where, assuming a deflection in the $x$-direction, $R_{22}$ is the $(2,2)$ element of the $6 \times 6$ transfer matrix describing the transformation of particle trajectory from 


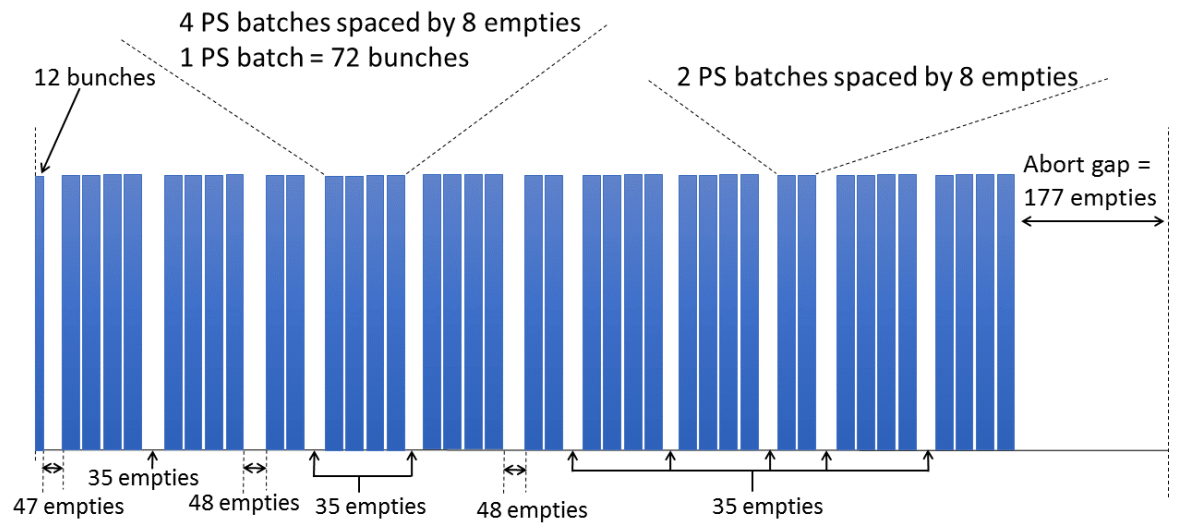

Figure 2: LHC bunch filling pattern from 2016. Bunch spacing $\sim 25$ ns. 2748 buckets out of the 3564 available are filled. Several different filling schemes are used for the LHC and are being considered for HL-LHC.

one crab cavity to the corresponding anti-crab cavity. Neglecting the transverse coupling and dispersion, the transfer matrix can be considered as a $2 \times 2$ matrix, where $R_{22}$ describes how an angular deflection at the first cavity transforms into an angular deflection at the second cavity. Because of the $\pi$ phase advance and similar values of beta functions at crab and anti-crab cavities, $R_{22}$ is close to -1 for the HL-LHC.

There are various gaps in the LHC bunch train arising from the rise times of the injection and extraction kickers for the proton synchrotron (PS), the SPS and the beam dump kicker of the LHC (abort gap). A representative bunch structure of the LHC bunch train is shown in Figure 2. Consequently there is strong transient beam loading in the accelerating RF cavities (as opposed to the crab cavities) as there is full beam loading during a PS batch of 72 bunches 70 (shown in blue) and zero beam loading during the gaps (shown in white). To keep the accelerating cavity amplitude and phase constant over a full train of bunches making a whole turn of the LHC in the presence of transient beam 
loading, the klystron forward power takes an amplitude and phase modulation as dictated by the Low Level RF controls (LLRF) that responds to the gaps. Charged bunches passing through a cavity change its amplitude and phase. Acceleration cavities are detuned in the presence of high beam currents acting in quadrature to the RF current in order to reduce the power demanded from the amplifiers (klystrons at the LHC for the accelerating cavities). Because the cavities are detuned, the power demanded then peaks during each gap in the bunch train. The LHC's detuning scheme was optimized at the outset so that the demand for klystron power during gaps does not exceed $300 \mathrm{~kW}$. This is achieved by detuning the cavity for half the peak beam current, hence the name "half detuning" [12, 13. The half-detuning scheme requires $200 \mathrm{~kW}$ average klystron power for the nominal LHC beam current ( 2808 bunches, $1.15 \times 10^{11}$ particles per bunch). After optimization of the coupling between klystron and the cavity, the power requirement scales linearly with the beam current. The beam current for HL-LHC is almost double the nominal LHC current (2748 bunches, $2.2 \times 10^{11}$ particles per bunch [1]), hence using the half detuning scheme, the required klystron average power will be near $400 \mathrm{~kW}$ and the peak power will be near to $600 \mathrm{~kW}$. This level exceeds the klystron saturation power of $300 \mathrm{~kW}$ installed prior to 2008. All the accelerating RF systems installed at this time were designed for a maximum of $300 \mathrm{~kW}$ continuous wave $(\mathrm{CW})$ operation. Increasing the RF power available for acceleration would require a significant modification of the RF power chain, including high voltage power supplies, klystrons, circulators, loads and input couplers [14]. To overcome the issue without major upgrades of the RF system, a new detuning scheme was proposed, tested in the LHC during 2016, and has been operationally available since 2017 [13, 15]. This scheme modulates the klystron phase but not its amplitude. The cavity amplitude is kept constant during the turn. As a consequence, bunch timing can no longer be perfectly maintained. The phase of bunches with respect to the RF clock progressively slip during the bunch train but then are finally recovered during the abort gap. With this scheme the klystron power is constant for the whole turn. The required klystron power is minimized by full detuning of the 
acceleration cavity for the average beam current. The full detuning scheme was into the cavity must still be adjusted for the peak klystron power. The level of coupling is specified by the external quality factor $Q_{e}$.

Perfect operation of the crab cavities (as opposed to the acceleration cavities) requires the $\mathrm{RF}$ field to be zero when the centroid of a bunch is at the centre $\mathrm{RF}$ clock then each crab cavity needs to have its RF phase adjusted during the bunch train. Without this adjustment rotation of the bunch envelope is not about its centre. The power requirements of a crab cavity is next to zero for a bunch that is on axis. The cavity power requirement increases with bunch The cavity power requirement stays relatively small for worst case, anticipated, beam offsets. The amplifier power requirement depends on the coupling of the cavity to the waveguide and is set for perfect transmission at the cavity when the cavity power requirement is maximum. As the power requirement for the crab cavities is modest the external quality factor $Q_{e}$ is quite large (small coupling).

The crab cavities for the HL-LHC are superconducting and consequentially have very large intrinsic quality factors $Q_{0}$. The loaded quality factor $Q_{L}$, is related to $Q_{0}$ and $Q_{e}$ by the relation

$$
\frac{1}{Q_{L}}=\frac{1}{Q_{0}}+\frac{1}{Q_{e}},
$$

which is large as both $Q_{0}$ and $Q_{e}$ are large. The time it takes to alter the phase of a cavity by one radian without the application of vast amounts of power is approximately equal to $Q_{L} \backslash \omega$. As the $Q_{L} \sim 10^{6}$ the crab cavity cannot be made to follow the 100 ps peak-to-peak phase modulation of the bunches arising from the full detuning LLRF control algorithm. Therefore the bunch centre arrives at the cavity early or late, and the transverse momentum kick is not zero at the bunch centre, resulting in an asymmetric kick. The kick received by the bunch centre results in a displacement as it passes the collision point. This is 


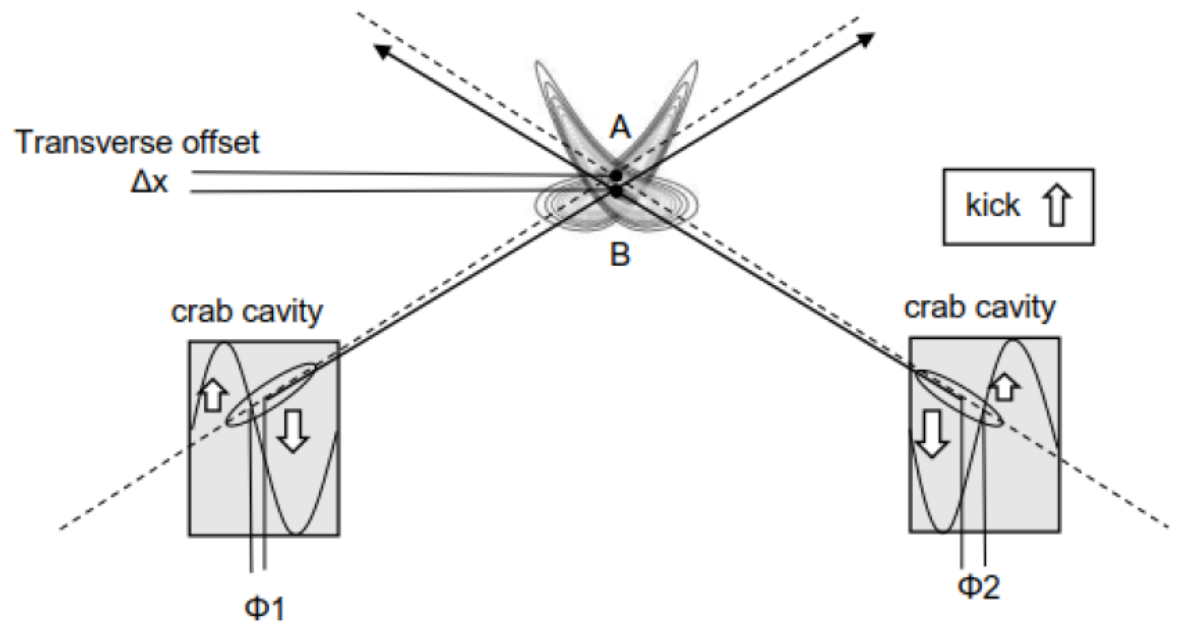

Figure 3: Schematic diagram of the collisions at the IP with coherent phase shifts in both colliding bunches.

illustrated in Figure 3. When the crab cavities are synchronized and for identical bunch phase shifts $\phi$ on the two beams then the bunch centres have a transverse displacement when they meet at the IP determined by

$$
\Delta x=\frac{c}{\omega} \frac{\theta}{2} \phi
$$

135 they have identical filling patterns and the acceleration cavities run the same control and detuning algorithms [15]. Therefore it will just displace the horizontal vertex. However, because HL-LHC bunches are very long with respect to the RF period (about 170 degrees for $4 \sigma_{z}$ ), this results in the crabbing kicks gaining a significant sinusoidal distortion (Figure 3). This distortion reduces peak luminosity. This paper analyses the luminosity reduction caused by phase modulation in the accelerating cavities [10].

Section 2 derives formulae for integrated peak luminosity with Gaussian bunch distributions including RF curvature. In section 3 particle tracking sim- 


\section{Analytical model of peak luminosity with RF phase modulation}

\subsection{Bunch distribution in the moving frame}

The horizontal transverse phase-space coordinate around the IP is defined by $\left(x, x^{\prime}\right)$. The focus is on horizontal momentum kicks from crab cavities. The hourglass effect is not considered in this study as the estimated luminosity reduction due to this effect is small [17].

Consideration is made of the transfer matrix between an initial position along the beam line, $S_{0}$, to a final position, $S_{1}$, which transforms the phase space coordinate $\left(x, x^{\prime}\right)$ as

$$
\left(\begin{array}{l}
x_{1} \\
x_{1}^{\prime}
\end{array}\right)=\left(\begin{array}{cc}
\sqrt{\frac{\beta_{1}}{\beta_{0}}}\left(\cos \mu+\alpha_{0} \sin \mu\right) & \sqrt{\beta_{1} \beta_{0}} \sin \mu \\
-\frac{\alpha_{1}-\alpha_{0}}{\sqrt{\beta_{0} \beta_{1}}} \cos \mu-\frac{1+\alpha_{0} \alpha_{1}}{\sqrt{\beta_{0} \beta_{1}}} \sin \mu & \sqrt{\frac{\beta_{0}}{\beta_{1}}}\left(\cos \mu-\alpha_{1} \sin \mu\right)
\end{array}\right)\left(\begin{array}{c}
x_{0} \\
x_{0}^{\prime}
\end{array}\right)
$$

155

where a transverse kick from the crab cavity has been included, $\delta x_{c . c}^{\prime}=-\frac{e V_{1}}{E_{s}} \sin \left(k z_{c . c}+\phi\right)$ where $k=\frac{\omega}{c}$ is the wave number from the crab cavity and $\mathrm{z}$ is the longitudinal position from the witness particle. As we assume a thin kick, we neglect $\delta x_{c . c}$. Hence one can express the phase space coordinates at the IP in terms of the phase space at the crab cavities as

$$
\begin{aligned}
x_{I P} & =\alpha_{c . c} \sqrt{\frac{\beta^{*}}{\beta_{c . c}}} x_{c . c}+\sqrt{\beta^{*} \beta_{c . c}} x_{c . c}^{\prime}-\sqrt{\beta^{*} \beta_{c . c}} \frac{e V_{1}}{E_{s}} \sin \left(k z_{c . c}+\phi\right) \\
x_{I P}^{\prime} & =-\frac{x_{c . c}}{\sqrt{\beta^{*} \beta_{c . c}}}
\end{aligned}
$$


In the following analytical solutions, the particle density of a bunch in both coordinates at the crab cavity is defined to be Gaussian using

$$
\rho_{c . c}\left(x_{c . c}, x_{c . c}^{\prime}, z_{c . c}\right)=\frac{\exp \left(-\frac{z_{c . c}^{2}}{2 \sigma_{z}^{2}}\right) \cdot \exp \left[-\left(\frac{\gamma_{c . c} x_{c . c}^{2}+2 \alpha_{c . c} x_{c . c} x_{c . c}^{\prime}+\beta_{c . c} x_{c . c}^{\prime}}{2 \varepsilon_{g}}\right)\right]}{(2 \pi)^{3 / 2} \sigma_{z} \varepsilon_{g}},
$$

where $\gamma=\left(1+\alpha^{2}\right) / \beta$ and $\varepsilon_{g}$ is the r.m.s geometrical transverse emittance. It should be noted that the Twiss parameters $\beta, \alpha$ and $\gamma$ are not mutually independent, this is a consequence of Liouville's Theorem and the conservation of emittance. By rearranging Eq. 8, one can obtain the phase space coordinate at the crab cavity in terms of the coordinate at the IP as

$$
\begin{aligned}
& x_{c . c}=-\sqrt{\beta^{*} \beta_{c, c}} x_{I P}^{\prime} \\
& x_{c . c}^{\prime}=\frac{x_{I P}}{\sqrt{\beta^{*} \beta_{c . c}}}+\alpha_{c . c} \sqrt{\frac{\beta^{*}}{\beta_{c . c}}} x_{I P}^{\prime}+\frac{e V_{1}}{E_{s}} \sin \left(k z_{c . c}+\phi\right) \\
& z_{c . c}=z_{I P}
\end{aligned}
$$

and substituting this into Eq. 9. the particle distribution at the IP can be expressed as

$$
\rho_{I P}\left(x_{I P}, x_{I P}^{\prime}, z_{I P}\right)=\frac{\exp \left(-\frac{z_{I P}^{2}}{2 \sigma_{z}^{2}}\right) \cdot \exp \left[-\left(\frac{x_{I P}^{2}+\beta^{* 2} x_{I P}^{\prime}{ }^{2}+C}{2 \sigma_{x}^{* 2}}\right)\right]}{(2 \pi)^{3 / 2} \sigma_{z} \varepsilon_{g}},
$$

where $\sigma_{x}^{*}$ is the horizontal beam size at the IP and

$$
C=\left(\beta^{*} \beta_{c . c} \frac{e V_{1}}{E_{s}} \sin \left(k z_{I P}+\phi\right)+2 \sqrt{\beta^{*} \beta_{c . c}} x_{I P}\right) \frac{e V_{1}}{E_{s}} \sin \left(k z_{I P}+\phi\right) .
$$

175 Now we integrate $\rho_{I P}\left(x_{I P}, x_{I P}^{\prime}, z_{I P}\right)$ over $x_{I P}^{\prime}$ to obtain the particle distri- 
bution in $x_{I P}$ and $z_{I P}$ as

$$
\begin{aligned}
\rho_{I P}\left(x_{I P}, z_{I P}\right) & =\frac{\exp \left(-\frac{z_{I P}^{2}}{2 \sigma_{z}^{2}}\right) \cdot \exp \left[-\left(\frac{x_{I P}^{2}+C}{2 \sigma_{x}^{* 2}}\right)\right]}{(2 \pi)^{3 / 2} \sigma_{z} \varepsilon_{g}} \int_{-\infty}^{\infty} \exp \left(\frac{-\beta^{* 2} x_{I P}^{\prime}{ }^{2}}{2 \sigma_{x}^{* 2}}\right) d x_{I P}^{\prime} \\
& =\frac{\exp \left(-\frac{z_{I P}^{2}}{2 \sigma_{z}^{2}}\right) \cdot \exp \left[-\left(\frac{x_{I P}^{2}+C}{2 \sigma_{x}^{* 2}}\right)\right]}{2 \pi \sigma_{z} \sigma_{x}^{*}}
\end{aligned}
$$

\subsection{Rotating the coordinate system at the IP}

The coordinate system of the bunch at the IP is rotated by half the crossing angle $\theta / 2$ into a new coordinate system, $\left(\tilde{x}_{I P}, \tilde{z}_{I P}\right)$; the counter-rotating bunch

be expressed in terms of $\left(x_{I P}, z_{I P}\right)$ as

$$
\left(\begin{array}{c}
x_{I P} \\
z_{I P}
\end{array}\right)=\left(\begin{array}{cc}
\cos \frac{\theta}{2} & -\sin \frac{\theta}{2} \\
\sin \frac{\theta}{2} & \cos \frac{\theta}{2}
\end{array}\right)\left(\begin{array}{l}
\tilde{x}_{I P} \\
\tilde{z}_{I P}
\end{array}\right)=\left(\begin{array}{l}
\tilde{x}_{I P} \cos \frac{\theta}{2}-\tilde{z}_{I P} \sin \frac{\theta}{2} \\
\tilde{x}_{I P} \sin \frac{\theta}{2}+\tilde{z}_{I P} \cos \frac{\theta}{2}
\end{array}\right) .
$$

Substituting this into Eq. 13 , one obtains

$\rho_{I P}^{(1)}\left(\tilde{x}_{I P}, \tilde{z}_{I P}\right)=\frac{\exp \left[-\frac{\left(\tilde{x}_{I P} \sin \frac{\theta}{2}+\tilde{z}_{I P} \cos \frac{\theta}{2}\right)^{2}}{2 \sigma_{z}^{2}}\right] \cdot \exp \left[-\frac{\left(\tilde{x}_{I P} \cos \frac{\theta}{2}-\tilde{z}_{I P} \sin \frac{\theta}{2}\right)^{2}+C_{1}}{2 \sigma_{x}^{* 2}}\right]}{2 \pi \sigma_{z} \sigma_{x}^{*}}$,

where

$$
\begin{aligned}
C_{1}= & \left\{\beta^{*} \beta_{c . c} \frac{e V_{1}}{E_{s}} \sin \left[k\left(\tilde{x}_{I P} \sin \frac{\theta}{2}+\tilde{z}_{I P} \cos \frac{\theta}{2}\right)+\phi_{1}\right]\right. \\
& \left.+2 \sqrt{\beta^{*} \beta_{c . c}}\left(\tilde{x}_{I P} \cos \frac{\theta}{2}-\tilde{z}_{I P} \sin \frac{\theta}{2}\right)\right\} \frac{e V_{1}}{E_{s}} \sin \left[k\left(\tilde{x}_{I P} \sin \frac{\theta}{2}+\tilde{z}_{I P} \cos \frac{\theta}{2}\right)+\phi_{1}\right] .
\end{aligned}
$$


For the counter-rotating bunches, the distribution becomes

$\rho_{I P}^{(2)}\left(\tilde{x}_{I P}, \tilde{z}_{I P}\right)=\frac{\exp \left[-\frac{\left(-\tilde{x}_{I P} \sin \frac{\theta}{2}+\tilde{z}_{I P} \cos \frac{\theta}{2}\right)^{2}}{2 \sigma_{z}^{2}}\right] \cdot \exp \left[-\frac{\left(\tilde{x}_{I P} \cos \frac{\theta}{2}+\tilde{z}_{I I} \sin \frac{\theta}{2}\right)^{2}+C_{2}}{2 \sigma_{x}^{* 2}}\right]}{2 \pi \sigma_{z} \sigma_{x}^{*}}$,

185

$$
\begin{aligned}
C_{2}= & \left\{\beta^{*} \beta_{c . c} \frac{e V_{1}}{E_{s}} \sin \left[k\left(-\tilde{x}_{I P} \sin \frac{\theta}{2}+\tilde{z}_{I P} \cos \frac{\theta}{2}\right)-\phi_{2}\right]\right. \\
& \left.-2 \sqrt{\beta^{*} \beta_{c . c}}\left(\tilde{x}_{I P} \cos \frac{\theta}{2}+\tilde{z}_{I P} \sin \frac{\theta}{2}\right)\right\} \frac{e V_{1}}{E_{s}} \sin \left[k\left(-\tilde{x}_{I P} \sin \frac{\theta}{2}+\tilde{z}_{I P} \cos \frac{\theta}{2}\right)-\phi_{2}\right] .
\end{aligned}
$$

\subsection{Bunch distribution in the non-deflecting transverse direction}

In the non-deflecting transverse direction, taken to be $y$, the particle distribution is not correlated to the longitudinal beam, thus the distribution at the IP can be expressed as

$$
\rho_{I P}\left(\tilde{y}_{I P}\right)=\frac{1}{\sqrt{2 \pi} \sigma_{y}^{*}} \exp \left(-\frac{\tilde{y}_{I P}^{2}}{2 \sigma_{y^{2}}^{*}}\right) .
$$

The nominal beam parameters of HL-LHC optics version 1.2 (HL-LHCV1.2) [18] are summarized in Table 2, which is used in the following calculations. The fullcrossing angle in the HL-LHCV1.2 optics is 510 prad.

Table 2: Twiss parameter of HL-LHC baseline optics version 1.2 (HL-LHCV1.2) 18. These Twiss parameters at the crab cavities are calculated by MADX [19].

\begin{tabular}{lcc}
\hline Parameter & $x$ & $y$ \\
\hline$\beta^{*}$ at IP5 $[\mathrm{m}]$ & $0.20^{a}$ & $0.20^{a}$ \\
$\alpha^{*}$ at IP5 & 0 & 0 \\
$\beta$ at C.C before IP5 $[\mathrm{m}]$ & 2453 & 2160 \\
$\alpha$ at C.C before IP5 & -14.0 & -36.7 \\
\hline
\end{tabular}

${ }^{a}$ For the baseline HL-LHCV1.3 optics [1, $\beta^{*}$ will be squeezed from $0.64 \mathrm{~m}$ to $0.15 \mathrm{~m}$ in the leveling scheme. 

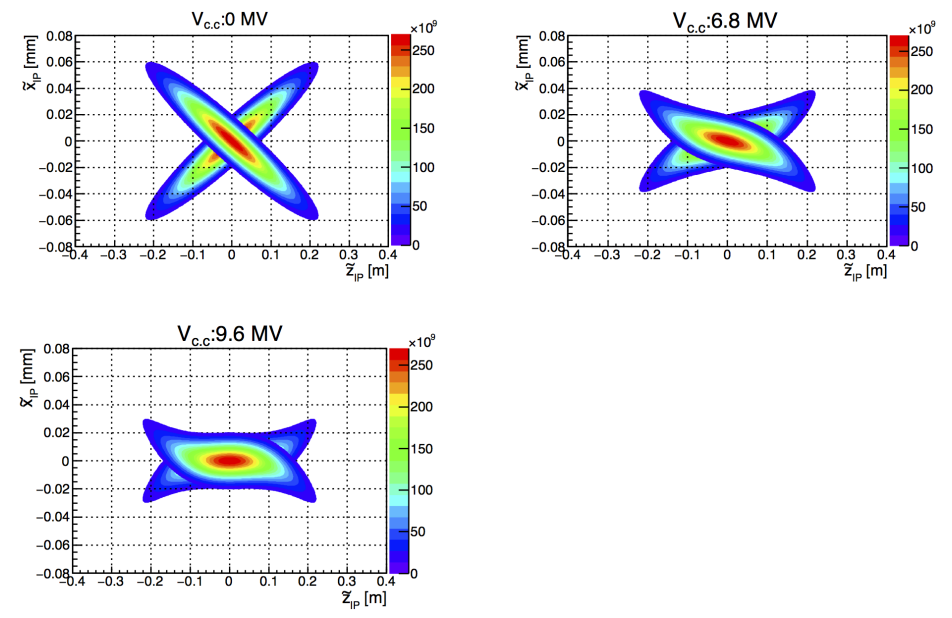

Figure 4: Bunch distributions of beam 1 and beam 2 at the IP without crab cavities (Top left), the baseline crabbing voltage of 6.8 MV (Top right) and 9.6 MV required for full compensation (Bottom left). In these plots, no phase modulation has been applied.

The total available crab voltage before the IP is planned to be at $6.8 \mathrm{MV}$, which is less than the total required crab voltage $\left(V_{1}\right)$ of $9.6 \mathrm{MV}$ for full correction of the crossing angle as given by Eq. 2. Full correction of the crossing angle gives too much luminosity for the current detectors and so installation of 9.6 MV is unnecessary at this stage. Figure 4 shows the distributions of two counterrotating bunches at the IP with the crab cavities operating with no crab voltage (Top left), at the nominal crab voltage of $6.8 \mathrm{MV}$ (Top right) and an unavailable crab voltage of 9.6 MV as required for full correction (Bottom left). The figure shows improved overlap densities of colliding bunches with crabbing. The partial compensation of the crossing angle with 6.8 MV as shown in Figure 4] provides sufficient luminosity for physics measurements by preventing pile-up when applying a $\beta^{*}$ leveling scheme [7, 8].

Bunch distributions at the IP for colliding bunches with identical phase shifts of $0.13,0.25,0.38$ and 0.50 radians at $400.79 \mathrm{MHz}$ are plotted with the nominal $6.8 \mathrm{MV}$ crab voltage in Figure 5 The phase shifts are identical when the counter rotating beams have identical filling schemes and the RF system runs an 

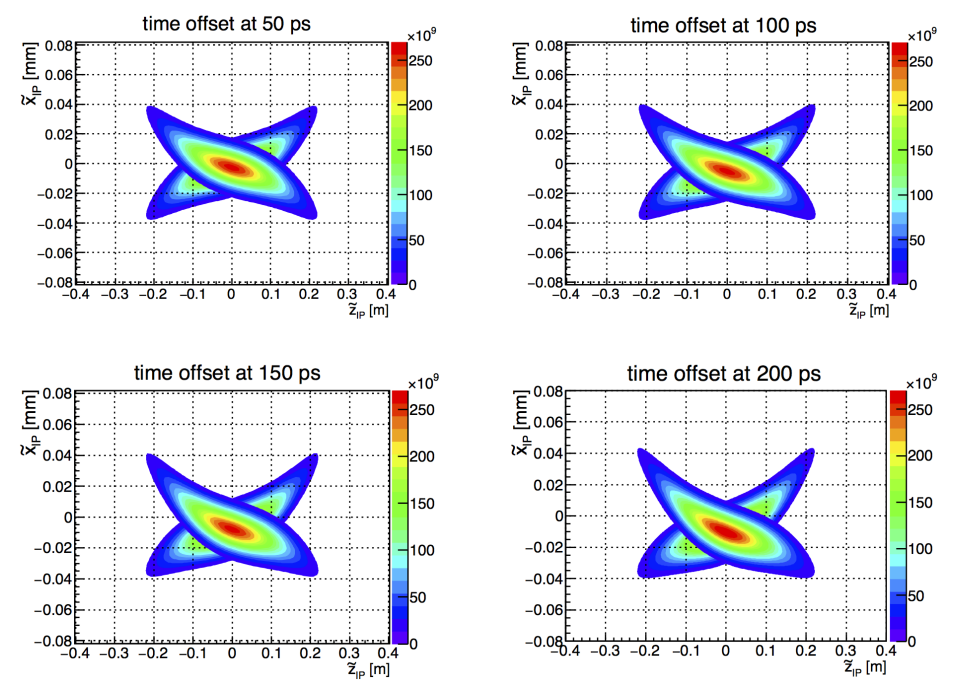

Figure 5: Bunch distributions of beam 1 and beam 2 at the IP with coherent phase modulations for the colliding pairs with total crab voltage at $6.8 \mathrm{MV}$. In these plots, the phase shifts are specified as a timing error.

identical full detuning algorithm. The transverse center of both counter-rotating bunches are displaced coherently from the IP with phase shifts as mentioned in Eq. 5 Furthermore, the sinusoidal distortion of bunches becomes stronger with the phase modulations.

\subsection{Luminosity calculation with phase modulations}

A peak luminosity [17] is obtained by the overlap integral of bunch densities with the longitudinal bunch positions. The bunches are translated longitudinally along the beam line $\left(z_{I P}\right)$ from the IP (where we define the time, $t=0$ ) to somewhere around the IP. The integral peak luminosity is then given by

$$
\begin{aligned}
& L=2 \cdot \cos ^{2} \frac{\theta}{2} N_{1} N_{2} f_{r e v} n_{b} \iiint \int_{-\infty}^{\infty} \rho_{I P}^{(1)}\left(\tilde{x}_{I P}, \tilde{z}_{I P},-c t\right) \cdot \rho_{I P}^{(1)}\left(\tilde{y}_{I P}\right) \cdot \\
& \rho_{I P}^{(2)}\left(\tilde{x}_{I P}, \tilde{z}_{I P}, c t\right) \cdot \rho_{I P}^{(2)}\left(\tilde{y}_{I P}\right) d \tilde{x}_{I P} d \tilde{y}_{I P} d \tilde{z}_{I P} d(c t) .
\end{aligned}
$$

In this equation, $N_{1}, N_{2}$ are the numbers of particles in the two counter-rotating bunches, $f_{r e v}$ is the revolution frequency and $n_{b}$ is the number of bunches in a 
beam. The term $2 \cdot \cos ^{2} \frac{\theta}{2}$ is the kinetic factor [20]. The longitudinal position of the translated bunch in the $z_{I P}$ coordinate can be written as $z_{I P} \pm c t$. Assuming identical transverse bunch size and bunch length for two colliding bunches, the peak luminosity can be written as

$$
\begin{aligned}
L= & \frac{\cos ^{2} \frac{\theta}{2} N_{1} N_{2} f_{r e v} n_{b}}{4 \pi^{3} \sigma_{z}^{2} \sigma_{x}^{* 2} \sigma_{y}^{* 2}} \iiint \int_{-\infty}^{\infty} \exp \left(-\frac{\tilde{y}_{I P}^{2}}{\sigma_{y}^{* 2}}\right) \cdot \\
& \exp \left[-\frac{\left(\tilde{x}_{I P} \cos \frac{\theta}{2}-\tilde{z}_{I P} \sin \frac{\theta}{2}\right)^{2}+\left(\tilde{x}_{I P} \cos \frac{\theta}{2}+\tilde{z}_{I P} \sin \frac{\theta}{2}\right)^{2}+\tilde{C}_{1}+\tilde{C}_{2}}{2 \sigma_{x}^{* 2}}\right] . \\
& \exp \left[-\frac{\left(\tilde{x}_{I P} \sin \frac{\theta}{2}+\tilde{z}_{I P} \cos \frac{\theta}{2}-c t\right)^{2}+\left(-\tilde{x}_{I P} \sin \frac{\theta}{2}+\tilde{z}_{I P} \cos \frac{\theta}{2}+c t\right)^{2}}{2 \sigma_{z}^{2}}\right] d \tilde{x}_{I P} d \tilde{y}_{I P} d \tilde{z}_{I P} d(c t),
\end{aligned}
$$

where $\tilde{C}_{1}$ and $\tilde{C}_{2}$ are given as

$$
\begin{aligned}
\tilde{C}_{1}= & \left\{\beta^{*} \beta_{c . c} \frac{e V_{1}}{E_{s}} \sin \left[k\left(\tilde{x}_{I P} \sin \frac{\theta}{2}+\tilde{z}_{I P} \cos \frac{\theta}{2}-c t\right)+\phi_{1}\right]\right. \\
& \left.+2 \sqrt{\beta^{*} \beta_{c . c}}\left(\tilde{x}_{I P} \cos \frac{\theta}{2}-\tilde{z}_{I P} \sin \frac{\theta}{2}\right)\right\} \frac{e V_{1}}{E_{s}} \sin \left[k\left(\tilde{x}_{I P} \sin \frac{\theta}{2}+\tilde{z}_{I P} \cos \frac{\theta}{2}-c t\right)+\phi_{1}\right] \\
\tilde{C}_{2}= & \left\{\beta^{*} \beta_{c . c} \frac{e V_{1}}{E_{s}} \sin \left[k\left(-\tilde{x}_{I P} \sin \frac{\theta}{2}+\tilde{z}_{I P} \cos \frac{\theta}{2}+c t\right)-\phi_{2}\right]\right. \\
& \left.-2 \sqrt{\beta^{*} \beta_{c . c}}\left(\tilde{x}_{I P} \cos \frac{\theta}{2}+\tilde{z}_{I P} \sin \frac{\theta}{2}\right)\right\} \frac{e V_{1}}{E_{s}} \sin \left[k\left(-\tilde{x}_{I P} \sin \frac{\theta}{2}+\tilde{z}_{I P} \cos \frac{\theta}{2}+c t\right)-\phi_{2}\right]
\end{aligned}
$$

Furthermore, we assume $N=N_{1}=N_{2}$ and integrate over $\tilde{y}_{I P}$, the integrated peak luminosity becomes

$$
\begin{aligned}
L= & \frac{\cos ^{2} \frac{\theta}{2} N^{2} f_{r e v} n_{b}}{4 \pi^{5 / 2} \sigma_{x}^{* 2} \sigma_{y}^{*} \sigma_{z}^{2}} \iiint_{-\infty}^{\infty} \exp \left[-\frac{\left(\tilde{x}_{I P} \cos \frac{\theta}{2}-\tilde{z}_{I P} \sin \frac{\theta}{2}\right)^{2}+\left(\tilde{x}_{I P} \cos \frac{\theta}{2}+\tilde{z}_{I P} \sin \frac{\theta}{2}\right)^{2}+\tilde{C}_{1}+\tilde{C}_{2}}{2 \sigma_{x}^{* 2}}\right] . \\
& \exp \left[-\frac{\left(\tilde{x}_{I P} \sin \frac{\theta}{2}+\tilde{z}_{I P} \cos \frac{\theta}{2}-c t\right)^{2}+\left(-\tilde{x}_{I P} \sin \frac{\theta}{2}+\tilde{z}_{I P} \cos \frac{\theta}{2}+c t\right)^{2}}{2 \sigma_{z}^{2}}\right] d \tilde{x}_{I P} d \tilde{z}_{I P} d(c t) .
\end{aligned}
$$


For the case of unequal horizontal and vertical bunch size and bunch length of colliding pairs, the peak luminosity can be written as

$$
\begin{aligned}
L= & \frac{\cos ^{2} \frac{\theta}{2} N^{2} f_{r e v} n_{b}}{\pi^{5 / 2} \sigma_{x 1}^{*} \sigma_{x 2}^{*} \sigma_{z 1} \sigma_{z 2} \sqrt{8\left(\sigma_{y 1}^{*}+\sigma_{y 2}^{*}{ }^{2}\right.}} \iiint_{-\infty}^{\infty} d \tilde{x}_{I P} d \tilde{z}_{I P} d(c t) \\
& \exp \left\{-\left[\frac{\left(\tilde{x}_{I P} \cos \frac{\theta}{2}-\tilde{z}_{I P} \sin \frac{\theta}{2}\right)^{2}+\tilde{C}_{1}}{2 \sigma_{x 1}^{*}{ }^{2}}+\frac{\left(\tilde{x}_{I P} \cos \frac{\theta}{2}+\tilde{z}_{I P} \sin \frac{\theta}{2}\right)^{2}+\tilde{C}_{2}}{2 \sigma_{x 2}^{*}{ }^{2}}\right]\right\} . \\
& \exp \left\{-\left[\frac{\left(\tilde{x}_{I P} \sin \frac{\theta}{2}+\tilde{z}_{I P} \cos \frac{\theta}{2}-c t\right)^{2}}{2 \sigma_{z 1}^{2}}+\frac{\left(-\tilde{x}_{I P} \sin \frac{\theta}{2}+\tilde{z}_{I P} \cos \frac{\theta}{2}+c t\right)^{2}}{2 \sigma_{z 2}^{2}}\right]\right\} .
\end{aligned}
$$

\section{Comparison of peak luminosity}

Particle tracking simulations have been made using PYTRACK [21]. Twiss parameters and first-order transfer maps (sector maps) of the HL-LHC optics have been calculated using MADX [19]. Using the linear maps, we track a single bunch with PYTRACK to compute a bunch distribution at the IP. Finally we numerically compute overlap peak luminosity and compare to the analytical model.

\subsection{Twiss parameters and sector-map from $M A D X$}

The ring optics of the HL-LHC round optics version 1.2 [18 $\left(\beta_{x}^{*}=\beta_{y}^{*}\right.$ at the IP1 and IP5) have been used for this simulation. The optics minimizes transverse beam size at these two IPs (minimal beta).

Sector maps have been created for IP1, two accelerating RF cavities per beam, $8 \mathrm{MV} /$ cav (the LHC accelerating RF is $16 \mathrm{MV} /$ beam during physics data taking) and IP5 with one pair of crab cavities, which gives rise to a horizontal momentum kick on colliding beams. Transfer matrices of each section are then calculated by MADX.

PYTRACK is a fast tracking code using first order transfer maps and special thin elements like crab cavities and beam-beam interactions. It is implemented 
in pure PYTHON programing code. The code tracks particles in a six dimensional phase space with coordinates: $\left(x, x^{\prime}, y, y^{\prime}, z, \Delta p_{z} / p_{z}\right)$ where $x^{\prime}=p_{x} / p_{z}$ and $y^{\prime}=p_{y} / p_{z}$, where $p_{x, y, z}$ are the horizontal, vertical and longitudinal momenta, $\Delta p_{z} / p_{z}$ is the fractional momentum deviation, and $z=c \Delta t$ the longitudinal position offsets with respect to the reference particle.

The colliding beams receive a transverse and longitudinal momentum kick at the crab cavities every turn (depending on their transverse position and phase relative to the cavity $\mathrm{RF}$ of the beam centroids), which can be expressed as follows:

$$
\begin{aligned}
\Delta x^{\prime} & =\frac{e V_{1}}{p_{z}} \sin \left(\phi_{s}-k z\right) \\
\frac{\Delta p_{z}}{p_{z}} & =-k \cdot x \cdot \frac{e V_{1}}{p_{z}} \cos \left(\phi_{s}-k z\right)
\end{aligned}
$$

where $\phi_{s}$ is the synchronous phase. In order to keep quasi-static synchrotron motion, the crab voltage is linearly ramped up over 1000 turns which corresponds to about two synchrotron periods (synchrotron frequency: $f_{s}=23.8 \mathrm{~Hz}$ ). Furthermore, in this simulation, the crabbing and anti-crabbing RF voltages at the crab cavities are set to be equal to the nominal crab voltage (6.8 MV).

Both colliding bunches are injected at IP1 where the Twiss parameter $\alpha$ equals zero and bunch distributions are observed at the IP5. The initial bunch consists of $10^{5}$ macroparticles which are generated with a Gaussian distribution in transverse phase space. The initial bunch size and angle are calculated by Twiss parameters at IP1 for both colliding bunches. As for the longitudinal bunch profile, two distributions are considered: Gaussian and q-Gaussian [1] as shown in Figure 6, generated by Beam Longitudinal Dynamics (BLonD) 22$]$ code. The longitudinal bunch profile in the current LHC is close to a q-Gaussian distribution. The two longitudinal profiles on Figure 6 have the same full width at half maximum (FWHM). A Gaussian with that FWHM will have a $\sigma_{z}=$ $9 \mathrm{~cm}$ (Table 1). Both longitudinal distributions are matched to a stationary RF bucket. Figure 7 shows the bunch distributions at IP5 for the case of Gaussian and q-Gaussian longitudinal bunch distributions, respectively. 

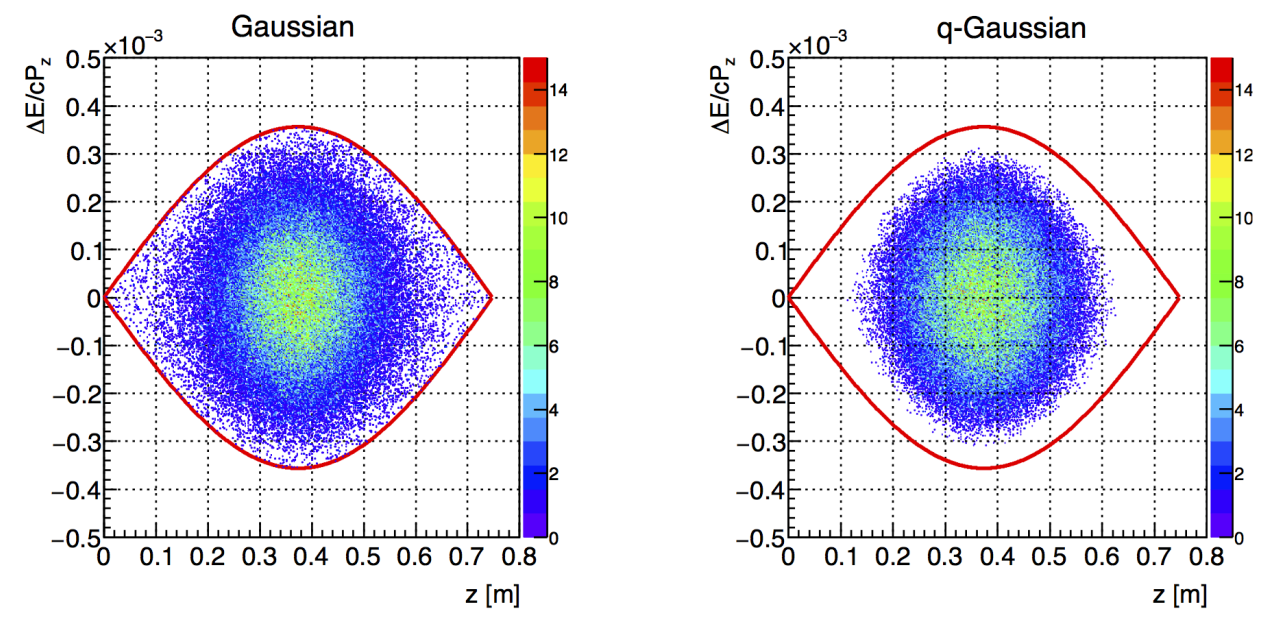

Figure 6: The initial bunch distributions: Gaussian and q-Gaussian, in longitudinal phase space. The red curves are the separatrix.
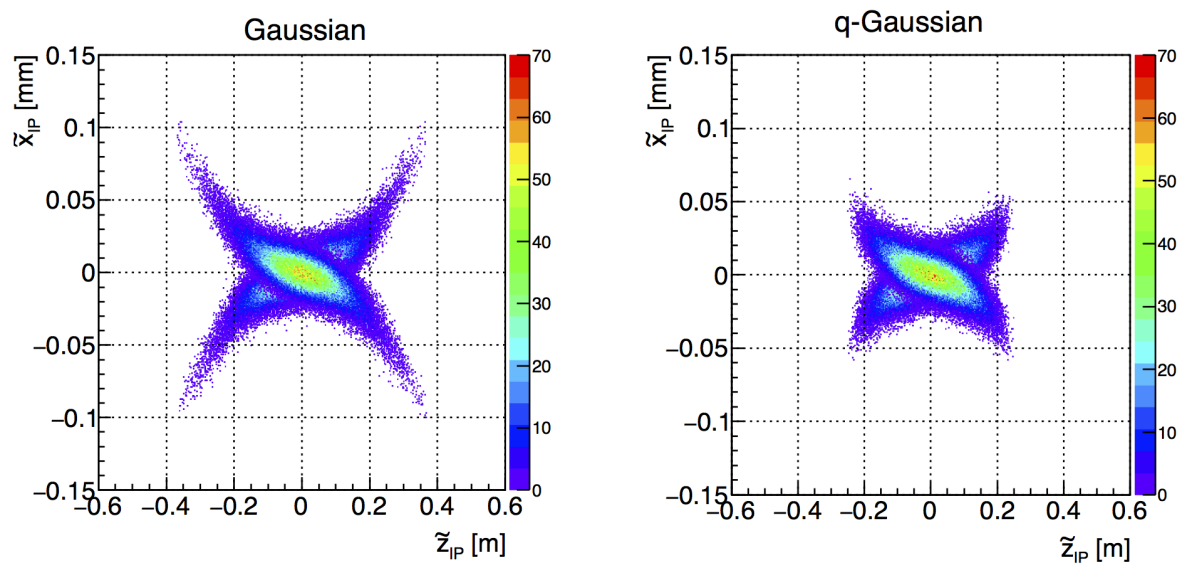

Figure 7: Bunch distributions at IP5 without phase offsets for both colliding beams. Left one indicates Gaussian and right one is q-Gaussian distributions with total crab voltage at $6.8 \mathrm{MV}$.

Figure 8 shows the bunch distributions at IP5 with coherent and incoherent phase modulations for the Gaussian and q-Gaussian longitudinal bunch profiles. We define a coherent phase error as a phase error that affects both counterrotating bunches equally. Given the identical filling pattern for both beams, 
the phase modulation should match for colliding pairs. For an incoherent phase

zero crossing angle and zero crab voltage), the purple dashed line shows the case of coherent phase modulations with the crab voltage of 9.6 MV required for head collisions (i.e. full compensation from the crab cavities), the green dashed line is the case of incoherent phase modulations with $9.6 \mathrm{MV}$ (modeled by applying 305 coherent phase modulations with the nominal crab voltage of $6.8 \mathrm{MV}$, the blue dashed line shows the case of incoherent phase modulations with $6.8 \mathrm{MV}$ and the black dashed line shows the luminosity without crab cavities. The reduction 

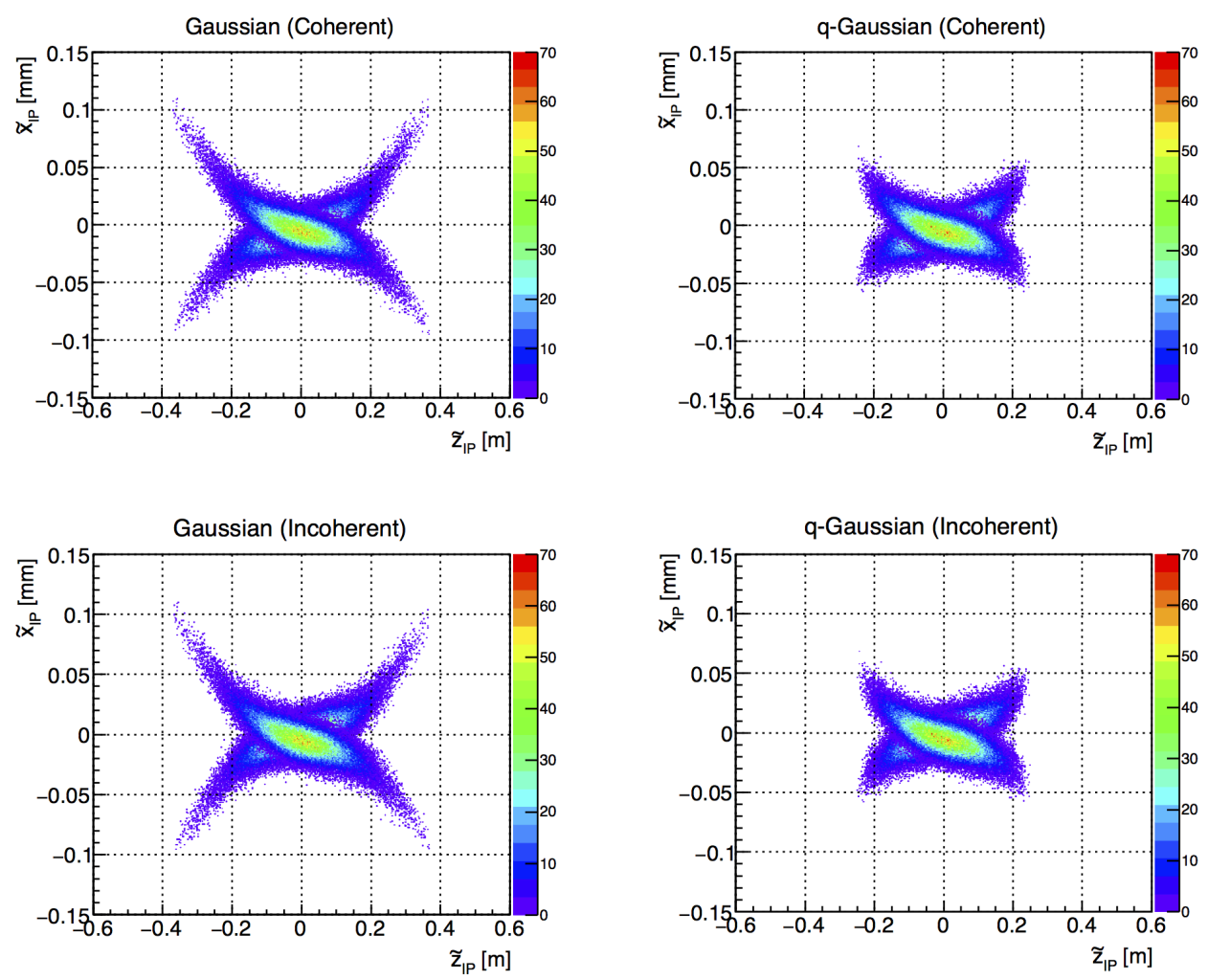

Figure 8: Bunch distributions at IP5 with coherent (Top) and incoherent (Bottom) phase offsets. Left plots are Gaussian and right ones are q-Gaussian longitudinal bunch profiles. In this figure, the total crab voltage is $6.8 \mathrm{MV}$.

factor $\left(R_{p}\right)$ is about 0.34 between the full crabbing compensation with no phase error and the no crabbing compensation, which is consistent with Eq. 1 . The red square dots and circles show the case of coherent phase modulations for Gaussian and q-Gaussian longitudinal bunch distribution respectively. The blue triangles and crosses show the case of incoherent phase modulations for Gaussian and q-Gaussian longitudinal bunch profiles.

For the case of coherent phase error, the simulation for the Gaussian longitudinal bunch profile predicts a smaller luminosity than the analytical model. This is due to the initial energy spread, which introduces a spread in betatron phase advance between the crab and anti-crab cavities; thus off-momentum par- 


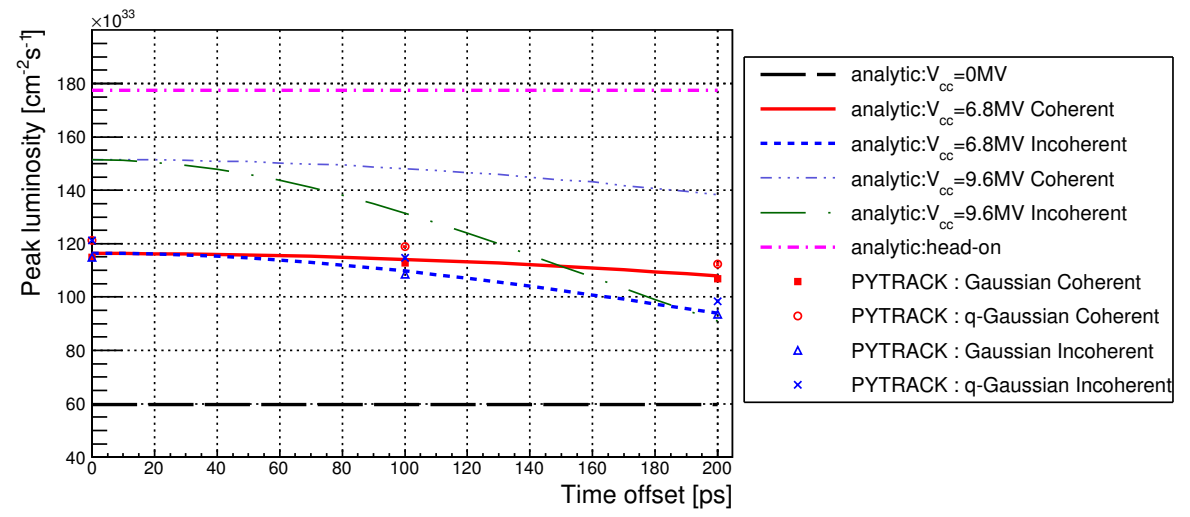

Figure 9: Peak luminosity vs crab cavity phase error.

ticles will experience a residual crabbing, reducing peak luminosity. To verify this, simulations have been run for the Gaussian longitudinal bunch profile with and without energy spread. Very good agreement has been seen between the simulation and the analytical model in the absence of an energy spread (Table 3p. In addition, the analytical model does not consider the effect of the main accelerating RF cavities as this would also introduce an energy spread,

it only considers a transverse momentum kick introduced by the crab cavities (Eq. 25).

Table 3: Simulation and analytical results for the reduction of peak luminosity rate with coherent phase errors for q-Gaussian and Gaussian longitudinal bunch distributions. The percentage drop in luminosity is given in parenthesis below each luminosity value.

\begin{tabular}{c|c|c|c|c}
\hline Time offset & \multicolumn{4}{|c}{ Peak luminosity $\left[10^{35} \mathrm{~cm}^{-2} s^{-1}\right]$} \\
\hline$[\mathrm{ps}]$ & Analytical & \multicolumn{2}{|c}{ Gaussian } & q-Gaussian \\
\hline & & $\left(\frac{\delta p}{p}=0\right)$ & $\left(\frac{\delta p}{p} \neq 0\right)$ & $\left(\frac{\delta p}{p} \neq 0\right)$ \\
\hline 0 & 1.163 & $1.160 \pm 1.1 \times 10^{-3}$ & $1.148 \pm 1.3 \times 10^{-3}$ & $1.211 \pm 1.0 \times 10^{-3}$ \\
\hline 100 & 1.141 & $1.140 \pm 1.1 \times 10^{-3}$ & $1.128 \pm 9.1 \times 10^{-4}$ & $1.189 \pm 6.6 \times 10^{-3}$ \\
& $(1.89 \%)$ & $(1.72 \pm 0.13 \%)$ & $(1.74 \pm 0.14 \%)$ & $(1.82 \pm 0.10 \%)$ \\
\hline 200 & 1.079 & $1.080 \pm 1.2 \times 10^{-3}$ & $1.068 \pm 1.2 \times 10^{-3}$ & $1.124 \pm 1.2 \times 10^{-3}$ \\
& $(7.22 \%)$ & $(6.90 \pm 0.14 \%)$ & $(6.97 \pm 0.15 \%)$ & $(7.18 \pm 0.13 \%)$ \\
\hline
\end{tabular}


On the other hand for the q-Gaussian longitudinal bunch distribution, the peak luminosity is larger than the analytical one. A q-Gaussian distribution is more localized at the centre of the RF bucket than a Gaussian distribution, resulting in an increased peak luminosity. The analytical model assumes a Gaussian distribution and therefore does not account for the effect of other distributions.

It is important to investigate incoherent phase errors as well as coherent errors to estimate the tolerance on uncorrelated jitter between the crab cavities and their dependence on peak luminosity. The results are summarized in Table. 4 and plotted in Figure 9 by the blue line and markers.

Table 4: Simulation and analytical results for reduction of peak luminosity rate with incoherent phase errors for q-Gaussian and Gaussian longitudinal bunch distributions. The percentage drop in luminosity is given in parenthesis below each luminosity value.

\begin{tabular}{c|c|c|c}
\hline Time offset & \multicolumn{3}{|c}{ Peak luminosity $\left[10^{35} \mathrm{~cm}^{-2} \mathrm{~s}^{-1}\right]$} \\
\hline$[\mathrm{ps}]$ & Analytical & Gaussian & q-Gaussian \\
\hline 0 & 1.163 & $1.148 \pm 1.3 \times 10^{-3}$ & $1.211 \pm 1.0 \times 10^{-3}$ \\
\hline 100 & 1.097 & $1.086 \pm 1.4 \times 10^{-3}$ & $1.146 \pm 1.3 \times 10^{-3}$ \\
& $(5.67 \%)$ & $(5.40 \pm 0.16 \%)$ & $(5.37 \pm 0.13 \%)$ \\
\hline 200 & 0.939 & $0.936 \pm 1.4 \times 10^{-3}$ & $0.985 \pm 7.5 \times 10^{-4}$ \\
& $(19.2 \%)$ & $(18.5 \pm 0.15 \%)$ & $(18.7 \pm 0.09 \%)$ \\
\hline
\end{tabular}

A larger reduction in peak luminosity is observed for incoherent phase errors than for coherent ones. This is because a coherent phase error will allow the bunches to collide without an offset, but with a distortion to the longitudinal bunch profile. An incoherent phase error will distort the longitudinal bunch profile, but will also introduce a transverse offset between the bunches at the IP.

\section{Conclusions}

The challenging nature of the high luminosity upgrade to the LHC carries technical risk. This risk is moderated by detailed analysis of every aspect of the 
new technologies and operating procedures. Key deliverables are integrated luminosity and levelled luminosity for optimal detector performance. This paper analyses how a new mode of operation for the RF system will impact luminosity. The scheme avoids a hugely expensive upgrade to the acceleration system. In this scheme klystron power is kept constant and only klystron phase is varied. The scheme is operated with the acceleration cavities, fully detuned for average beam current. The scheme has been tested and it has been operationally available since July 2017. The avoidance of varying klystron power to compensate for transient beam-loading results in a modulation of bunch phase through the bunch train with respect to the RF clock. For this scheme it is expected that the maximum peak-to-peak phase displacement will be $\sim 0.25 \mathrm{rad}$ at $400 \mathrm{MHz}$ (100 ps in time) along a bunch train. The RF phase of the crab cavities cannot be modulated to follow this phase modulation due to their high loaded quality factors $Q_{L}$, thus individual bunches see a different crabbing phase, the ideal being zero. This phase error enhances bunch distortions at the IP as a result of the sinusoidal variation of the RF and hence reduces peak luminosity. To evaluate the luminosity reduction, the analytical model for the peak luminosity including the effect of phase modulations on the crab cavities has been derived. It has been validated using particle tracking simulations with MADX and PYTRACK.

365 The difference between Gaussian and q-Gaussian longitudinal distributions has been investigated. The tracking simulations and the analytical model are in good agreement with each other for both coherent and incoherent phase modulations for the crab cavity scheme. The analytical model assumes a Gaussian distribution with no longitudinal momentum spread and disparities between the model and different simulation conditions are explained.

For the expected maximum coherent phase error of $100 \mathrm{ps}$, the reduction in peak luminosity is about $1.89 \%$ for a Gaussian distribution based on the analytical model. The expected maximum incoherent phase error of $100 \mathrm{ps}$ (not planned), will cause a luminosity reduction of about $5.67 \%$.

The peak luminosity is leveled down to $5 \times 10^{34} \mathrm{~cm}^{-2} \cdot \mathrm{s}^{-1}$ [1] in order to reduce the number of pile up events at the IPs, which can otherwise prevent 
the ATLAS and CMS detectors from resolving different events. For the leveling scheme, it is concluded that the impact of the full-detuning RF control scheme is negligible for both coherent and incoherent phase errors and well within the tolerance for baseline HL-LHC operation. Understanding how luminosity depends on RF parameters is important for machine operation.

\section{Acknowledgements}

The authors would like to thank Dr. H. Timko for assistance with BLonD code, and Dr. R. Tomas and Mr. L. Medina for helpful comments and discussions. The HiLumi LHC Design Study is included in the High Luminosity LHC project and is partly funded by the European Commission within the Framework Program 7 Capacities Specific Program, Grant Agreement 284404. This work has also been funded by the UK research council STFC through the grant HL-LHC-UK (ST/N001575/1) and Cockcroft Institute (ST/P002056/1).

\section{References}

[1] E. Metral, et al., 'Update of the HL-LHC operational scenarios for proton operation', CERN-ACC-NOTE-2018-0002 (2018) pp. 1-41.

[2] S. Fartoukh, A. Valishev, Y. Papaphilippou, D. Shatilov, 'Compensation of the long-range beam-beam interactions as a path towards new configurations for the high luminosity LHC', Phys. Rev. ST Accel. Beams 18 (2015) pp. 121001. doi:10.1103/PhysRevSTAB.18.121001.

[3] J. Wenninger, 'Approaching the Nominal Performance at the LHC', Proceedings of 8th International Particle Accelerator Conference (IPAC'17) (2017) pp. 13-18. doi:10.18429/JACoW-IPAC2017-MOYAA1.

[4] R. B. Palmer, 'Energy scaling, crab crossing and the pair problem', SLAC Report No. SLAC-PUB-4707 (1988) pp. 1-7. 
[5] B. Xiao, L. Alberty, S. Belomestnykh, I. Ben-Zvi, R. Calaga, C. Cullen, O. Capatina, L. Hammons, Z. Li, C. Marques, J. Skaritka, S. Verdú-Andres, $\mathrm{Q}$. Wu, 'Design, prototyping, and testing of a compact superconducting double quarter wave crab cavity', Phys. Rev. ST Accel. Beams 18 (2015) pp. 041004. doi:10.1103/PhysRevSTAB.18.041004.

[6] A. Dexter, G. Burt, R. Apsimon, 'Active lower order mode damping for the four rod LHC crab cavity', Nuclear Instruments and Methods in Physics Research Section A 844 (2017) pp. 62-71. doi:10.1016/j.nima.2016.11. 021.

[7] G. Apollinari, I. Béjar Alonso, O. Brüning, M. Lamont, L. Rossi, 'HighLuminosity Large Hadron Collider (HL-LHC) : Preliminary Design Report', CERN-2015-005, FERMILAB-DESIGN-2015-02, (2015). doi:10. 5170/CERN-2015-005

[8] J. P. Koutchouk, 'Luminosity Optimization and Leveling', Proceedings of Chamonix 2010 workshop on LHC performance (2010) pp. 342-347.

[9] M. Hostettler, A. Calia, K. Fuchsberger, M. Gabriel, G. Hemelsoet, M. Hruska, D. Jacquet, J. Wenninger, ' $\beta$ * levelling using the LHC Lumi Server (MD 2427)', CERN-ACC-NOTE-2018-0001 (2018) pp. 1-10.

[10] S. Yi-Peng, R. Assmann, J. Barranco, T. Rogelio, T. Weiler, F. Zimmermann, R. Calaga, A. Morita, 'beam dynamics aspects of crab cavities in the CERN Large Hadron Collider', Phys. Rev. ST Accel. Beams 12 (2009) pp. 101002. doi:10.1103/PhysRevSTAB.12.101002.

[11] B. Yee, 'Crab cavity voltage and luminosity calculation', EuCARD-NOT425 2010-008 (2010) pp. 1-26.

[12] P. Baudrenghien, 'The Tuning Algorithm of the LHC 400 MHz Superconducting Cavities', CERN-AB-2007-011 (2007) pp. 1-11. 
[13] P. Baudrenghien, T. Mastoridis, 'Proposal for an RF roadmap towards ultimate intensity in the LHC', Proceedings of International Particle Accelerator Conference (IPAC'12) (2012) pp. 154-156.

[14] R. Calaga, HL-LHC RF Roadmap, Proceedings of LHC Performance Workshop CERN-ATS-2015-002 (2014) pp. 209-216. doi:10.5170/ CERN-2015-002.209

[15] T. Mastoridis, P. Baudrenghien, J. Molendijk, 'Cavity voltage phase modulation to reduce the high-luminosity Large Hadron Collider RF power

1 requirements', Phys. Rev. Accel. Beams 20 (2017) pp. 101003. doi: 10.1103/PhysRevAccelBeams.20.101003

[16] D. Boussard, 'RF Power Requirements for a High Intensity Proton Collider', Proceedings of Particle Accelerator Conference (PAC'91) (1991) pp. $2447-2449$.

[17] W. Herr, B. Muratori, 'Concept of Luminosity', CAS (2003) pp. 361-378.

[18] R. Tomas, L. Medina, 'Parameter update for the nominal HL-LHC: Standard, BCMS, and 8b+4e, (HL-LHC Parameters v6.1.0') (2017).

[19] MAD - Methodical Accelerator Design Web Home, available at http://mad.web.cern.ch/mad/.

[20] C. Moller, 'General properties of the characteristic matrix in the theory of elementary particles I', K. Danske Vidensk. Selsk. Mat.-Fys. Medd. 23 (1945) pp. 1-48.

[21] R. Calaga, R. de Maria, R. Miyamoto, E. Yamakawa, PYTRACK: Particle transport code.

[22] H. Timko, H. Muller, J, A. Lasheen, D. Quartullo, 'Benchmarking the beam longitudinal dynamics code BLonD', Proceedings of 7th Int. Parti-

口 cle Accelerator Conf.(IPAC'16) (2016) pp. 3094-3097. doi:10.18429/ JACOW-IPAC2016-WEPOY045 
${ }_{455}$ [23] Python official site, available at https://www.python.org/. 